NISTIR 5053

Relative Permittivity Measurement of Square Copper-Lánininated Substrates Using the Full-Sheet Resonance Technique

Richard L. Lewis

QC

100

.456

N0.5053 



\section{Relative Permittivity Measurement of Square Copper-Laminated Substrates Using the Full-Sheet Resonance Technique}

Richard L. Lewis

Electromagnetic Fields Division Electronics and Electrical Engineering Laboratory National Institute of Standards and Technology Boulder, Colorado 80303-3328

January 1997

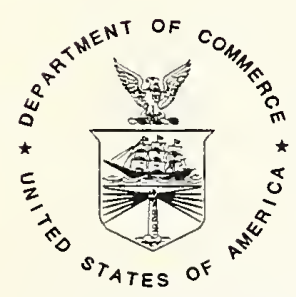

U.S. DEPARTMENT OF COMMERCE, Michael Kantor, Secretary TECHNOLOGY ADMINISTRATION, Mary L. Good, Under Secretary for Technology NATIONAL INSTITUTE OF STANDARDS AND TECHNOLOGY, Arati Prabhakar, Director 



\title{
Relative Permittivity Measurement of Square Copper-Laminated Substrates Using the Full-Sheet Resonance Technique
}

\author{
Richard L. Lewis \\ Electromagnetic Fields Division \\ National Institute of Standards and Technology \\ Boulder, Colorado 80303-3328
}

\begin{abstract}
A measurement program has been undertaken at NIST to evaluate the full-sheet resonance (FSR) technique, from which consistent relative permittivity values have been obtained. Here, we present an analysis of the theory underlying the FSR technique, along with a theoretical formulation described in the literature for related measurements, which when implemented with the FSR technique, should improve the technique's absolute measurement accuracy. A theoretical uncertainty analysis is presented both for the FSR technique assuming these improvements have been implemented and for the standard FSR technique. Numerical uncertainty estimates are presented for the standard FSR technique. Our measured results are compared against re-entrant cavity measurements of the substrate material, and both the FSR and re-entrant cavity measurements agree within expected uncertainty limits. We also present FSR measurement results for a circular disk, fed at the center of the disk, which tend to be substantiated by the re-entrant cavity measurements.
\end{abstract}

Key words: dielectric constant; FSR; full-sheet resonance; measurement error; relative permittivity; square panels; theoretical analysis; uncertainty analysis

\section{Introduction}

A number of authors have described a convenient method for measuring the relative permittivity of dielectric substrates having copper-clad top and bottom surfaces [1-5]. This method, known as the full-sheet resonance technique for measuring microwave circuit board substrates, determines the dielectric substrate's relative permittivity $\epsilon_{\mathrm{r}}^{\prime}$ by measuring the resonant frequencies of the resulting parallel plate cavity. Nondestructive measurement techniques such as FSR are important to the microwave communications industry where knowledge of substrate permittivity is critical to maintaining competitiveness. The technique is independent of substrate thickness, but is not capable of evaluating the uniformity of the relative permittivity over the substrate. A measurement program has been undertaken at NIST to evaluate the FSR technique using an automatic network analyzer (ANA), and results to date demonstrate that consistent values for the 
relative permittivity are obtained. Here, details of our implementation of the FSR method along with an associated uncertainty analysis are presented.

\section{Measurement Configuration}

Primarily, thin dielectric substrates having open sides and copper-clad top and bottom surfaces were tested. The entire panel acts as a resonant cavity, so by measuring the panel's resonant frequencies we obtain the average relative permittivity of the substrate. Since multiple resonant modes occur within the cavity, construction of a mode table is necessary to identify the particular mode excited at a given resonant frequency, which in turn requires keeping an accurate count of the resonant excitation frequencies encountered. The presence of closely spaced modes increases the difficulty of keeping an accurate resonant frequency count. Consequently, although the number of possible resonant modes within a panel is limitless, only the lower order modes are useful for determining relative permittivity.

Two different panel geometries were used in our study. In one case we measured transmission (the $S_{12}$ scattering-matrix parameter) through square panels, while in the other case we measured reflection (or $S_{11}$ ) from circular disk panels fed at the center of the circle. We also measured $S_{11}$ for circular disk panels after the open side had been metalized using copper tape. The test procedure using square panels is nondestructive when full panels are tested, while the procedure using circular disks is destructive because it requires use of a punch and die to stamp out a circle, drilling a small hole in the center of the circle for a coaxial connector's center conductor, and then soldering a coaxial connector to the copper sheets. Coupling between the ANA and the circular disks was optimized by connecting each circular disk directly to the operating port of the ANA using a short plug-to-plug type SMA connector, thereby shifting the transmission line resonances to very high frequencies.

The square panels are coupled to the ANA as shown in Figure 1 using two APC-7 $7 \mathrm{~mm}$ precision coaxial connectors positioned at opposite corners of the panel [1]. Transmission path coupling had to be optimized in order to maintain an accurate count of the modal excitation frequencies. Sufficiently enhanced coupling was obtained by maintaining direct contact between the center conductor of one coaxial connector and the panel's upper copper sheet and direct contact between the inside of the outer conductor of the opposite coaxial connector and the panel's lower copper sheet. The coaxial connectors were also turned so that they directly faced each other. Small alignment deviations led to missed resonances and in some cases to false resonances at low frequencies. Expected resonances going unobserved have previously been reported [1,2], whereas spurious low frequency resonances could be attributed to an increase in the reactive coupling between the coaxial connector and the panel which resonated with the panel. 


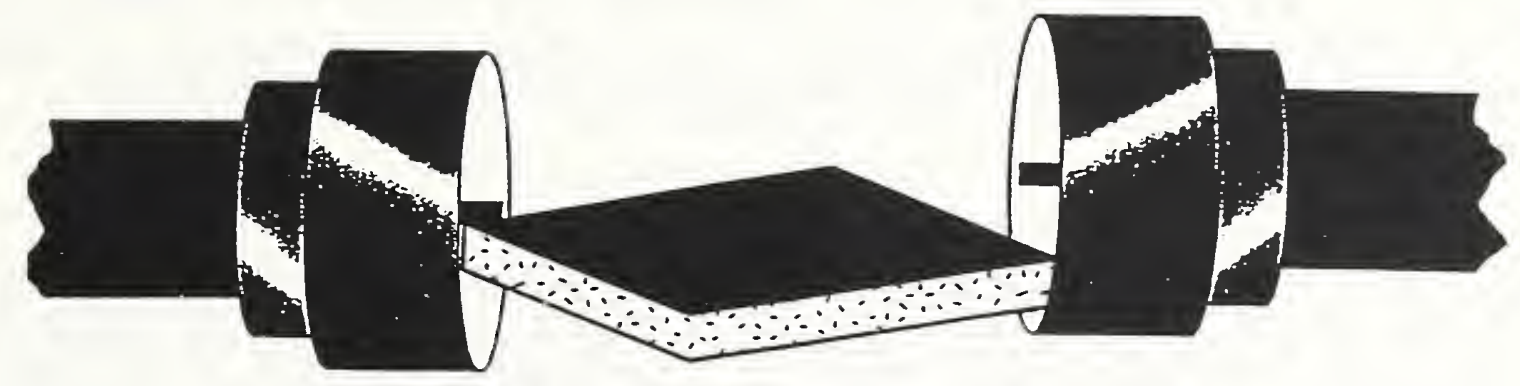

Figure 1. Coupling schematic between $7 \mathrm{~mm}$ coaxial connectors and a copper-clad dielectric substrate. 


\section{Analytical Formulation}

The relative permittivity of the substrate in a rectangular FSR panel is obtained from the resonant frequencies of an ideal cavity having perfectly conducting electric walls above and below the dielectric substrate and perfect magnetic walls along the sides [1]. This gives the expression

$$
\epsilon_{\mathrm{r}}^{\prime}=\left(\frac{\mathrm{c}}{2 \mathrm{f}_{\mathrm{mn}}}\right)^{2}\left[\left(\frac{\mathrm{m}}{\mathrm{x}_{1}}\right)^{2}+\left(\frac{\mathrm{n}}{\mathrm{y}_{1}}\right)^{2}\right],
$$

where $\mathrm{c}$ is the speed of light, $\mathrm{x}_{1}$ and $\mathrm{y}_{1}$ are the length and width of the panel (for a square panel, $\left.x_{1}=y_{1}\right), m$ and $n$ are integer-value mode numbers, and $f_{m n}$ is the resonant frequency of the ideal cavity corresponding to the $\mathrm{m} \mathrm{x} \mathrm{n}{ }^{\text {th }} \mathrm{TM}_{\mathrm{mno}}$ mode (electric field vector perpendicular to the conducting plates).

In practice, the resonant frequencies of a real cavity differ from their ideal values due to conductivity and radiation losses, electric or magnetic field perturbation at the cavity coupling points [3], and to overcoupled dual resonant modes [6]. When the FSR panel's side aspect ratio is nearly (but not exactly) equal to the square root of the ratio of two integers, then some modes can have their resonant peaks widened, skewed, or doubled [5]. Also, closely spaced resonances can have their resonant peaks distorted [5] by smearing. Resonances such as these may be used for mode counting but are not useful otherwise. However, provided the FSR panel shape is not a square, an unambiguous low order mode can be chosen [4, fig. 3] for a FSR permittivity measurement.

In the case of a totally closed cavity with conducting walls, Collin [7] derived a correction to the cavity's ideal resonant frequency $\mathrm{f}^{\prime}{ }_{\mathrm{mn}}$ in terms of such a cavity's metalized-wall conductivity quality factor $\mathrm{Q}_{\mathrm{C}}{ }_{\mathrm{C}}[2]$, obtaining

$$
\mathrm{f}_{\mathrm{mn}}^{\prime}=\left[1+\frac{1}{\mathrm{Q}_{\mathrm{C}}^{\prime}}\right]^{\frac{1}{2}} \mathrm{f}_{\mathrm{M} 0}^{\prime}
$$

where $\mathrm{f}_{\mathrm{M} 0}^{\prime}$ is the (unloaded) resonant frequency of the enclosed cavity when coupling per perturbation is disregarded. Equation (2) was obtained using a modal-series expansion, where each mode in the series corresponds to an eigenvalue solution for the field that would exist in a cavity with perfectly conducting walls. Collin [7] noted that a cavity having a combination of perfect electric and perfect magnetic walls could similarly be modeled using modal fields. We have formulated an analysis similar to Collin's to determine frequency pulling due to both finite conductivity and radiation loss in an open-sided FSR cavity, obtaining 


$$
\mathrm{f}_{\mathrm{mn}}=\left[1+\frac{1}{\mathrm{Q}_{\mathrm{C}}}+\frac{\mathrm{B}_{\mathrm{R}} / \mathrm{G}_{\mathrm{R}}}{\mathrm{Q}_{\mathrm{R}}}\right]^{\frac{1}{2}} \mathrm{f}_{\mathrm{M} 0},
$$

where $Q_{C}$ is the open cavity's conductivity quality factor for the metalized sides, $Q_{R}$ the radiation quality factor [8], $G_{R}+j B_{R} \equiv Y_{R}$ the normalized aperture admittance at the open side wall of the cavity, and $\mathrm{f}_{\mathrm{M} 0}$ the cavity's unloaded resonant frequency when coupling perturbation is disregarded. Equation (3) readily follows from Collin's derivation of eq (2) [7, section 7.7 through section 7.9] by replacing Collin's pure "short-circuit" modes with hybrid "short-circuit open-circuit" modes.

Based on analysis of the wave reflected from the end of a parallel plate waveguide assuming an extended dielectric slab [9], we expect the aperture susceptance of the open side wall of the cavity to be capacitive. Consequently $B_{R}$ will be positive, and in the case of separation distance between the top and bottom conducting sheets much less than a tenth of a wavelength, as considered here, we expect [10] $B_{R} / G_{R}>1$. Moreover, the magnitude of the reflection coefficient from the end of a dielectric-free parallel-plate waveguide [10] is $|r|=e^{-k t / 2}$, where $t$ is the guide width and $k=2 \pi / \lambda$. For typical panel thickness and frequencies under investigation, this would make $|\Gamma|$ close to unity, the character of a fairly good magnetic wall. Moreover, with a dielectric substrate the electric field will concentrate within the dielectric, making the aperture an even better magnetic wall.

The unloaded quality factor $\mathrm{Q}_{0}$ of a resonant cavity (lossless dielectric case) is obtained [8] by combining the cavity's conductivity and radiation quality factors

$$
\frac{1}{Q_{0}}=\frac{1}{Q_{C}}+\frac{1}{Q_{R}}
$$

The total or loaded quality factor $\mathrm{Q}_{\ell}$ of the system combines the cavity's internal quality factor $\mathrm{Q}_{0}$ with an external quality factor $\mathrm{Q}_{\mathrm{C}}$ representing external losses including coupling loss. The loaded quality factor is given [11] by

$$
\frac{1}{\mathrm{Q}_{\ell}}=\frac{1}{\mathrm{Q}_{0}}+\frac{1}{\mathrm{Q}_{\mathrm{e}}}=\frac{1+\kappa}{\mathrm{Q}_{0}},
$$

where $\mathrm{K}$ is the cavity coupling coefficient. If we assume an external series impedance $R_{e}+j X_{e}$ is connected to the cavity, then the measured resonant frequency $f_{M}$ of the total system will be given [12] by 


$$
\mathrm{f}_{\mathrm{M}}=\left[1+\frac{\kappa \mathrm{X}_{\mathrm{e}}}{2 \mathrm{Q}_{0} \mathrm{R}_{\mathrm{e}}}\right] \mathrm{f}_{\mathrm{M} 0},
$$

Kajfez [13] describes a "fractional linear curve fitting" procedure which enables all the unknown quantities in eqs (5) and (6) to be determined from ANA measurements. Earlier procedures [14, 15] for obtaining eq (5) parameters are more suited to small coupling coefficients, large quality factors, and the absence of interfering modes.

A theoretical formulation for the conductivity quality factor of a rectangular FSR is given by Taber [16], who obtained

$$
\mathrm{Q}_{\mathrm{C}}=\frac{\pi \mu_{0} \mathrm{f}_{\mathrm{M}} \mathrm{t}}{\mathrm{R}_{\mathrm{s}}}
$$

where $\mu_{0}$ is free-space permeability, $t$ the thickness of the dielectric substrate, and $R_{s}$, the surface resistance (including surface roughness [17]) of the metalized sides. Assuming a lossless dielectric, $Q_{R}$ can be determined from eqs (4), (5), and (7). Consequently, we can obtain a corrected value for $f_{m n}$ in eq (3) provided we can estimate $B_{R} / G_{R}$. To a first approximation, this ratio can be estimated using a theoretical formulation [9] corresponding to the simpler case of an extended dielectric slab, although a theoretical analysis similar to earlier work $[10,18]$ for the case where the dielectric substrate terminates at the end of the guide would be preferable since this would match the FSR configuration under study. Such an analysis should produce a theoretical value for $Q_{R}$ (for instance, an expression for $Q_{R}$ is given in [19] for a circular disk). It may be noted that eq (7) shows $Q_{C}$ is proportional to $t$, whereas $Q_{R}[16]$ is inversely proportional to t. Once $\mathrm{Q}_{\mathrm{R}}$ is determined theoretically we no longer need assume a lossless dielectric and the dielectric loss tangent can be obtained from the formula $\tan \delta=1 / Q_{0}-1 / Q_{C}-1 / Q_{R}$. Measured FSR quality factors are typically around 100 with relative uncertainties greater than $3 \%$; consequently the relative uncertainty in computing tan $\delta$ could be around $\pm 5 \%$ if the substrate's loss tangent is around $10^{-2}$ and around $\pm 50 \%$ if the loss tangent is around $10^{-3}$.

In addition to resonant frequency pulling, electric-field fringing beyond the dielectric substrate can lower the measured relative permittivity. This systematic error can be corrected by multiplying eq (1) by the idealized parallel-plate capacitance $\epsilon_{\mathrm{r}}^{\prime}\left(\mathrm{C}+\mathrm{C}_{\mathrm{f}}\right)$ and dividing by the actual capacitance $\epsilon_{\mathrm{r}}^{\prime} \mathrm{C}+\mathrm{C}_{\mathrm{f}}$, where $\mathrm{C}=\epsilon_{\mathrm{o}}\left(\mathrm{x}_{1} \mathrm{y}_{1} / \mathrm{t}\right), \epsilon_{\mathrm{o}}$ is free-space permittivity, and $\mathrm{C}_{\mathrm{f}}$ is the fringing capacitance of the panel. The relative permittivity is then obtained iteratively using

$$
\dot{\epsilon}_{\mathrm{r}}^{\prime}=\frac{\mathrm{C}+\mathrm{C}_{\mathrm{f}}}{\mathrm{C}+\mathrm{C}_{\mathrm{f}} / \epsilon_{\mathrm{r}}^{\prime}}\left(\frac{\mathrm{c}}{2 \mathrm{f}_{\mathrm{mn}}}\right)^{2}\left[\left(\frac{\mathrm{m}}{\mathrm{x}_{1}}\right)^{2}+\left(\frac{\mathrm{n}}{\mathrm{y}_{1}}\right)^{2}\right] \text {, }
$$


where $\dot{\epsilon}_{\mathrm{r}}^{\prime}$ is an improved value for the permittivity and the initial value $\epsilon_{\mathrm{r}}^{\prime}$, is obtained from eq (1). Assigning a value to $\mathrm{C}_{\mathrm{f}}$ is problematical, although as an approximation Kirchhoff's formula [20] for the fringe capacitance of two parallel circular disks could be used.

\section{Uncertainty Analysis}

The preceding formulation enables eq (1) to be corrected for some of the systematic error associated with the FSR technique. If the coupling coefficient $\kappa$ and the fringing capacitance $\mathrm{C}_{\mathrm{f}}$ are not evaluated, there will be commensurate increased uncertainties in the determination of $\epsilon_{r}^{\prime}$. We contrast the expected uncertainty using the preceding section's formulation versus the expected uncertainty using the much simpler (noniterative) expression

$$
\epsilon_{\mathrm{r}}^{\prime}=\left(\frac{\mathrm{c}}{2 \mathrm{f}_{\mathrm{M}}}\right)^{2}\left[\frac{\left(\mathrm{m} / \mathrm{x}_{1}\right)^{2}+\left(\mathrm{n} / \mathrm{y}_{1}\right)^{2}}{1+1 / \mathrm{Q}_{\ell}}\right]
$$

Table 1 presents expressions for some of the relative partial standard uncertainties [21, 22] in $\epsilon_{\text {r. }}^{\prime}$ The partial uncertainties in the middle column are associated with eq (9), while those in the column on the right represent the formulation of the preceding section. Each entry is obtained using the formula [21] $\frac{1}{\epsilon_{\mathrm{r}}^{\prime}} \frac{\partial \epsilon_{\mathrm{r}}}{\partial \mathrm{x}_{\mathrm{i}}} \Delta \mathrm{x}_{\mathrm{i}}$, where $\mathrm{x}_{\mathrm{i}}$, is a partial uncertainty component and $\Delta \mathrm{x}_{\mathrm{i}}$ is its uncertainty. Here, n.a. denotes not applicable, $\hat{X}_{e} \equiv X_{e} / R_{e}$, and $\overline{\Delta Q_{0}} \equiv\left(\frac{B_{R}}{G_{R}}-1\right) \frac{Q_{0}}{Q_{R}}+\Delta Q_{0}$. Typically [19], $\mathrm{Q}_{0}<\mathrm{Q}_{\mathrm{C}}<\mathrm{Q}_{\mathrm{R}}$, so neither component of $\overline{\Delta \mathrm{Q}_{0}}$ should be excessively dominant.

Table 2 presents estimated FSR uncertainties for our square panel measurements. Our estimates were formed using $Q_{0} \approx 100$ and $\kappa \approx 0.65$, agreeing in part with some extrinsic measurements. Also, we let $\left|\hat{X}_{\mathrm{e}}\right| \approx 3.5$. The combined standard uncertainty is the root sum of squares of the partial standard uncertainties [21]. Two partial uncertainties mentioned earlier, "coupling perturbation" and "adjacent resonances," are included in Table 2. Typically, lower results are generated for $\epsilon_{\mathrm{r}}^{\prime}$ by open-sided FSR panel measurements than by other techniques [5], partly due to fringing at the open panel sides and partly due to coupling perturbation, which decreases $\epsilon_{\mathrm{r}}^{\prime}$ by about $2 \%$ [3]. Table 2 accounts for this by skewing the combined uncertainty.

The FSR measurements were checked by measuring relative permittivity using a coaxial reentrant cavity, for which an uncertainty analysis is forthcoming [23]. In this technique, a circular dielectric sample is placed within a gap in the center conductor. A pair of coupling probes inside the cavity is used for a transmission measurement [8] of the resonant frequency and quality factor of the excited TEM mode, from which the sample's relative permittivity is determined. Table 3 gives a list of major re-entrant cavity uncertainties. For thin substrates, relative sample-thickness uncertainty dominates. In Table 3, the estimated accuracy for optimum-size samples agrees with estimates for similar instruments [20,24]. Sample thickness uncertainty is about $0.0076 \mathrm{~mm}$ ( $0.3 \mathrm{mil})$, augmented by $\sqrt{2}$ to account for differencing clad and unclad sample thicknesses, 
resulting in a relative thickness uncertainty for a $0.38 \mathrm{~mm}(15 \mathrm{mil})$ sample of $0.3 \sqrt{2} / 15$. The combined uncertainty reflects our sample's small thickness.

Table 1. Relative uncertainty analysis for $\epsilon_{\mathrm{r}}$.

\begin{tabular}{|c|c|c|}
\hline $\begin{array}{l}\text { Uncertainty } \\
\text { source }\end{array}$ & $\begin{array}{l}\text { No coupling } \\
\text { correction }\end{array}$ & $\begin{array}{l}\text { Fringing \& } \\
\text { coupling } \\
\text { corrected }\end{array}$ \\
\hline Frequency $\left(f_{M}\right)$ & $2 \frac{\Delta f_{M}}{f_{M}}\left|\frac{k \hat{X}_{e}}{Q_{0}}\right|$ & $2 \frac{\Delta f_{M}}{f_{M}}$ \\
\hline Quality factor $\left(\mathrm{Q}_{0}\right)$ & 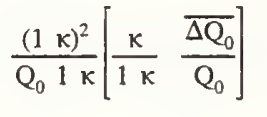 & $\left(\frac{1 k \hat{\mathrm{X}}_{e}}{\mathrm{Q}_{0} 0.5 k \hat{\mathrm{X}}_{c}}\right) \frac{\overline{\Delta \mathrm{Q}_{0}}}{\mathrm{Q}_{0} 1}$ \\
\hline Coupling factor $(\kappa)$ & n.a. & $\frac{1}{\mathrm{Q}_{0} 1} \frac{\hat{\mathrm{X}}_{\mathrm{e}}}{\mathrm{Q}_{0} 0.5 \kappa \hat{\mathrm{X}}_{\mathrm{e}}} \mid \frac{\Delta \kappa}{1 \mathrm{\kappa}}$ \\
\hline External impedance $\left(\hat{\mathrm{X}}_{\mathrm{e}}\right)$ & n.a. & $\frac{\kappa \Delta \hat{\mathrm{X}}_{\mathrm{c}}}{\mathrm{Q}_{0} 0.5 \kappa \hat{\mathrm{X}}_{\mathrm{e}}}$ \\
\hline Side length $\left(\mathrm{x}_{1}\right)$ & $2 \frac{\Delta x_{1}}{x_{1}} \frac{\left(\epsilon_{\mathrm{r}}^{\prime} 1\right) C_{\mathrm{r}}}{\epsilon_{\mathrm{r}}^{\prime} \mathrm{C} \quad 0.5 C_{\mathrm{r}}}$ & $2 \frac{\Delta x_{1}}{x_{1}}$ \\
\hline Fringing capacitance $\left(\mathrm{C}_{\mathrm{f}}\right)$ & n.a. & $\frac{C\left(\epsilon_{\mathrm{r}}^{\prime}\right) \Delta \mathrm{C}_{\mathrm{r}}}{\left(\epsilon_{\mathrm{r}}^{\prime} \mathrm{C} \mathrm{C}_{\mathrm{r}}\right)\left(\mathrm{C} \mathrm{C}_{\mathrm{r}}\right)}$ \\
\hline
\end{tabular}


Table 2. Relative-permittivity uncertainty analysis using FSR technique.

\begin{tabular}{lcc}
\hline $\begin{array}{l}\text { Uncertainty } \\
\text { source }\end{array}$ & $\begin{array}{c}\text { No coupling } \\
\text { correction }\end{array}$ & $\begin{array}{c}\text { Fringing \& } \\
\text { coupling } \\
\text { corrected }\end{array}$ \\
\hline Frequency & $4 \%$ & $2 \%$ \\
Quality factor & $1.6 \%$ & $0.6 \%$ \\
Coupling factor & n.a. & $0.8 \%$ \\
External impedance & n.a. & $0.8 \%$ \\
Side length (fringing) & $1.2 \%$ & $0.6 \%$ \\
Fringing capacitance & n.a. & $1 \%$ \\
Coupling perturbation & $2 \%$ & $1 \%$ \\
Adjacent resonances & $1 \%$ & $1 \%$ \\
Combined uncertainty & $+7 \%,-3 \%$ & $+4 \%,-2 \%$ \\
\hline
\end{tabular}

Table 3. Relative-permittivity uncertainty analysis using re-entrant cavity.

\begin{tabular}{lcc}
\hline \multicolumn{1}{c}{ Uncertainty } & & Uncertainty \\
\hline Sample thickness (thin [0.38 mm] sample) & & $2.83 \%$ \\
Optimum-sample uncertainty: & neg. & \\
\hline A) Sample thickness & $?$ & \\
B) Sample diameter & $?$ & \\
C) Resonant frequency & $?$ & $1 \%$ \\
D) Cavity dimensions & $?$ & \\
E) Air gap & & \\
F) Cavity quality factor (Q) & \\
Optimum-sample accuracy (similar instr.) & & \\
& \\
Combined re-entrant cavity uncertainty (based on small sample thickness): & $\pm 3 \%$. \\
\hline
\end{tabular}




\section{Specific Computational Expressions}

The square-panel substrate's relative permittivity is evaluated from ANA transmission measurements (see eq (9)) using

$$
\epsilon_{\mathrm{r}}^{\prime}=\left(\frac{\mathrm{c}}{2 \mathrm{x}_{1} \mathrm{f}_{\mathrm{M}}}\right)^{2}\left[\frac{\mathrm{m}^{2}+\mathrm{n}^{2}}{1+1 / \mathrm{Q}_{\ell}}\right]
$$

To a very good approximation $[11,12]$, the loaded quality factor $\mathrm{Q}_{\ell}$ in eq $(10)$ is determined using

$$
\mathrm{Q}_{\ell} \simeq \frac{\mathrm{f}_{\mathrm{M}}}{\mathrm{BW}}
$$

where BW is the measured half-power bandwidth. The relative permittivity of the open-sided circular disk is obtained by assuming a $\mathrm{TM}_{\text {moo }}$ mode [8] inside the dielectric filled cavity and enforcing the boundary condition $\mathrm{H}_{2}=0$ at the edge of the disk, resulting in

$$
\epsilon_{\mathrm{r}}^{\prime}=\frac{\left(\frac{\mathrm{cj}_{\mathrm{mo}}^{\prime}}{2 \pi \mathrm{r}_{1} \mathrm{f}_{\mathrm{M}}}\right)^{2}}{\left(1+\frac{1}{\mathrm{Q}_{\ell}}\right)}
$$

where $j^{\prime}{ }_{m 0}$, is the $(m+1)$ th zero of the derivative of the Bessel function of order zero [25] and $r_{1}$ is the radius of the disk. For the closed disk (copper-taped side), we also obtain the relative permittivity by assuming a $\mathrm{TM}_{\text {moo }}$ mode, but now the boundary condition $\mathrm{E}_{\mathrm{z}}=0$ is enforced [8] at the edge of the disk, producing

$$
\epsilon_{\mathrm{r}}^{\prime}=\frac{\left(\frac{c j_{\mathrm{mo}}}{2 \pi \mathrm{r}_{1} \mathrm{f}_{\mathrm{M}}}\right)^{2}}{\left(1+\frac{1}{\mathrm{Q}_{\ell}}\right)}
$$

where $j_{\mathrm{m} 0}$ is the $\mathrm{m}^{\text {th }}$ zero of the Bessel function of order zero [25]. 


\section{Analysis of Measurement Results}

Figure 2 presents FSR measurement results for a $25.4 \mathrm{~cm}$ square (10 in $\mathrm{x} 10$ in) panel with a $0.38 \mathrm{~mm}(15 \mathrm{mil})$ thick dielectric substrate having a nominal relative permittivity around 2.5 . Similarly, Figure 3 presents results for a $27.9 \mathrm{~cm}$ square (11 in x 11 in) panel with a $0.41 \mathrm{~mm}$ (16 mil) thick dielectric substrate having a nominal relative permittivity around 3.8. Confidence interval lines above and below the average relative permittivity are shown, corresponding to the uncorrected-case combined uncertainty in Table 2. Re-entrant cavity results are also shown, along with error bars based on Table 3 .

Typical measured $Q_{\ell}$ values were over 100 for the Figure 2 panel and under 50 for the Figure 3 panel. Also, efforts to enhance coupling may have resulted in critical over-coupled modes [11], in which case values for $\kappa>1$ would result. Our analytical uncertainty estimates suggest contracting the confidence interval lines around the average measured value in Figure 2 and expanding the confidence interval lines in Figure 3. Nevertheless, the uncertainty stated in Table 2 is generous enough to include the re-entrant cavity measurement within the uncertainty range in both figures and definitive coupling estimation is unavailable, so a single estimate is adopted to fit both cases.

The low datum point in Figure 2 near $1.5 \mathrm{GHz}$ and the low datum point in Figure 3 near $0.8 \mathrm{GHz}$ are attributed to interference by adjacent modes. Overall, the re-entrant cavity results and the FSR results are comparable within each procedure's experimental error. Increased FSR panel coupling has been reported [3] to result in reduced values for the relative permittivity, which may have contributed to the measurement discrepancy between the FSR and re-entrant cavity results in Figure 3. Moreover, as discussed in [5], high-permittivity material is more susceptible to FSR measurement errors than low permittivity material due to fringing, so agreement between FSR and re-entrant cavity results could be expected to be better for figure 2 than for figure 3 .

Figure 4 presents FSR results for two circular disks produced from the same material as described for the Figure 3 square panel. Results shown correspond to an open side $6.99 \mathrm{~cm}$ (2.75 in) diameter disk, an open side $12.7 \mathrm{~cm}$ (5.0 in) diameter disk, and a closed side (copper taped) $12.7 \mathrm{~cm}$ diameter disk. Figure 3 results are presented for comparison. The closed disk cavity was impaired by low quality factors and overcoupling with the result that its relative permittivity measurements were unreliable, particularly at the lower frequencies where the loaded quality factors were lowest. Nevertheless, the results shown in Figure 4 for the $12.7 \mathrm{~cm}$ closed disk demonstrate that the closed and open disk's resonant frequencies interlace each other, thus supporting the underlying analysis for eqs (12) and (13). Although not shown, similar interlacing between closed and open disk resonant frequencies occurred for the smaller $(6.99 \mathrm{~cm})$ disk.

The circular disk excitation coincides with the disk's resonant-mode field structure, so coupling perturbation error should be negligible. In figure 4, the discontinuities above $18 \mathrm{GHz}$ in the open disk plots are due to interference by adjacent modes accompanied by lowered quality factors. Except at these discontinuities, the open disk resonances were well isolated. With some 


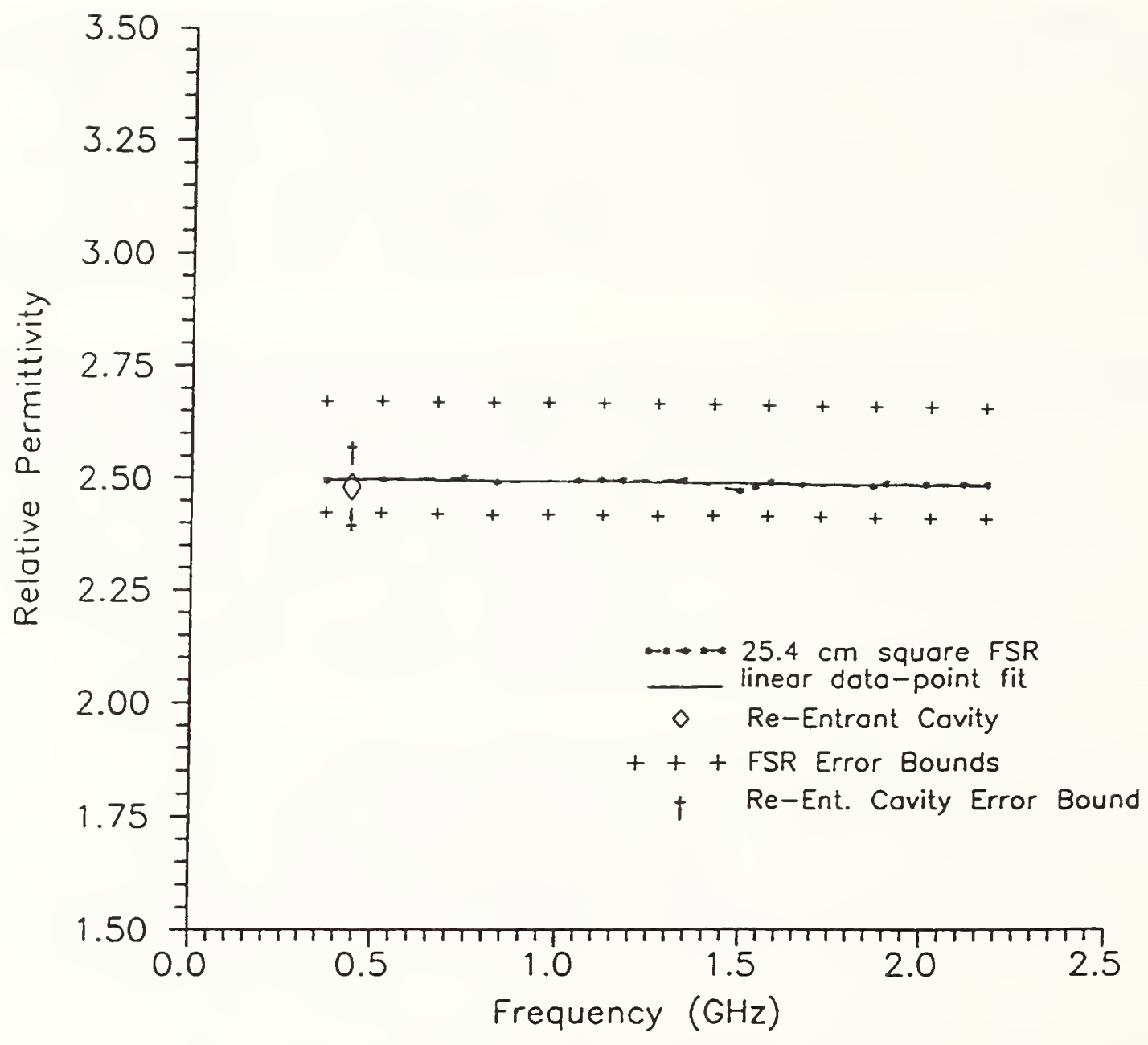

Figure 2. FSR permittivity results for $0.38 \mathrm{~mm}$ panel $25.4 \mathrm{~cm}$ square. 


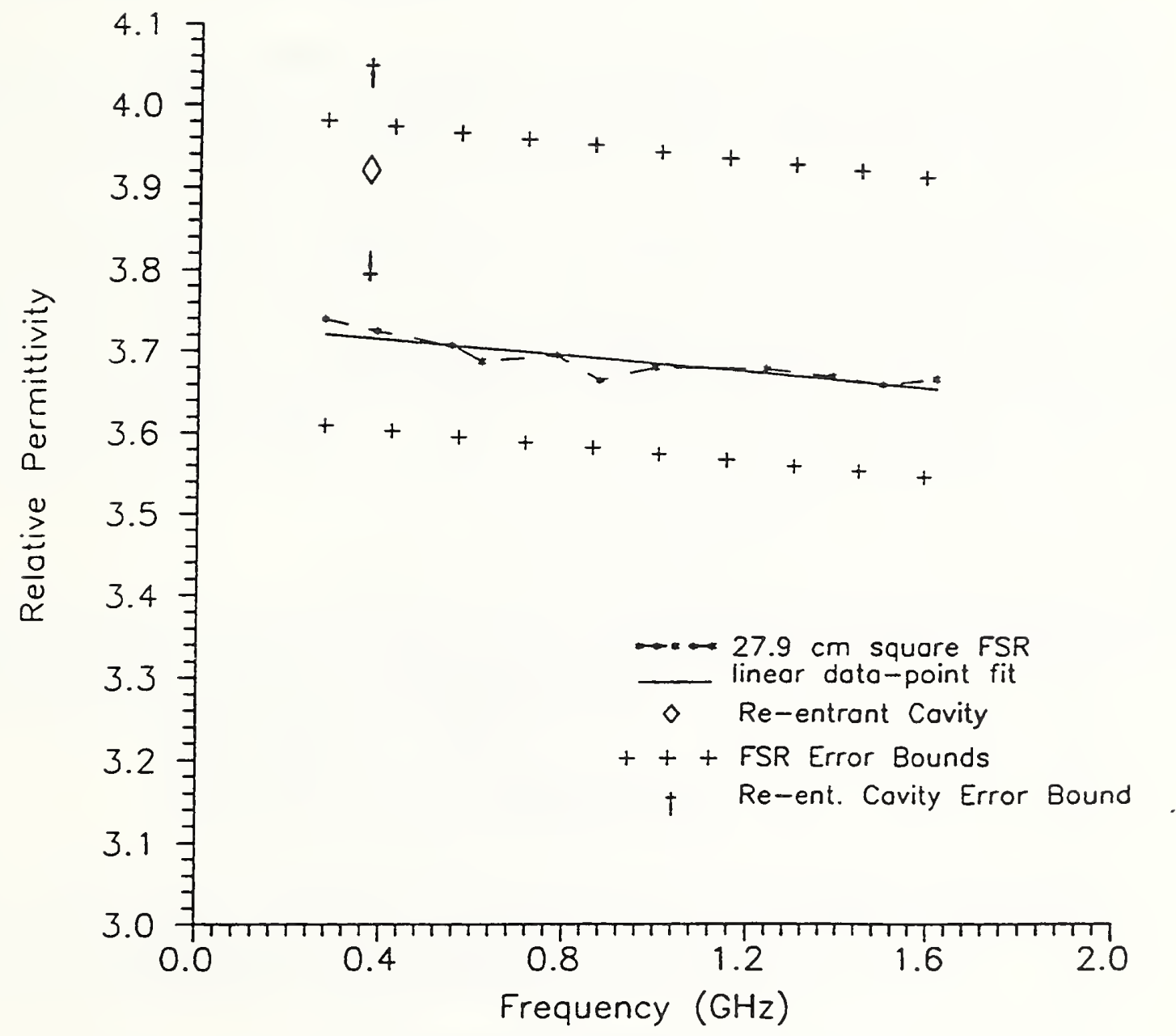

Figure 3. FSR permittivity results for $0.41 \mathrm{~mm}$ panel $27.9 \mathrm{~cm}$ square. 


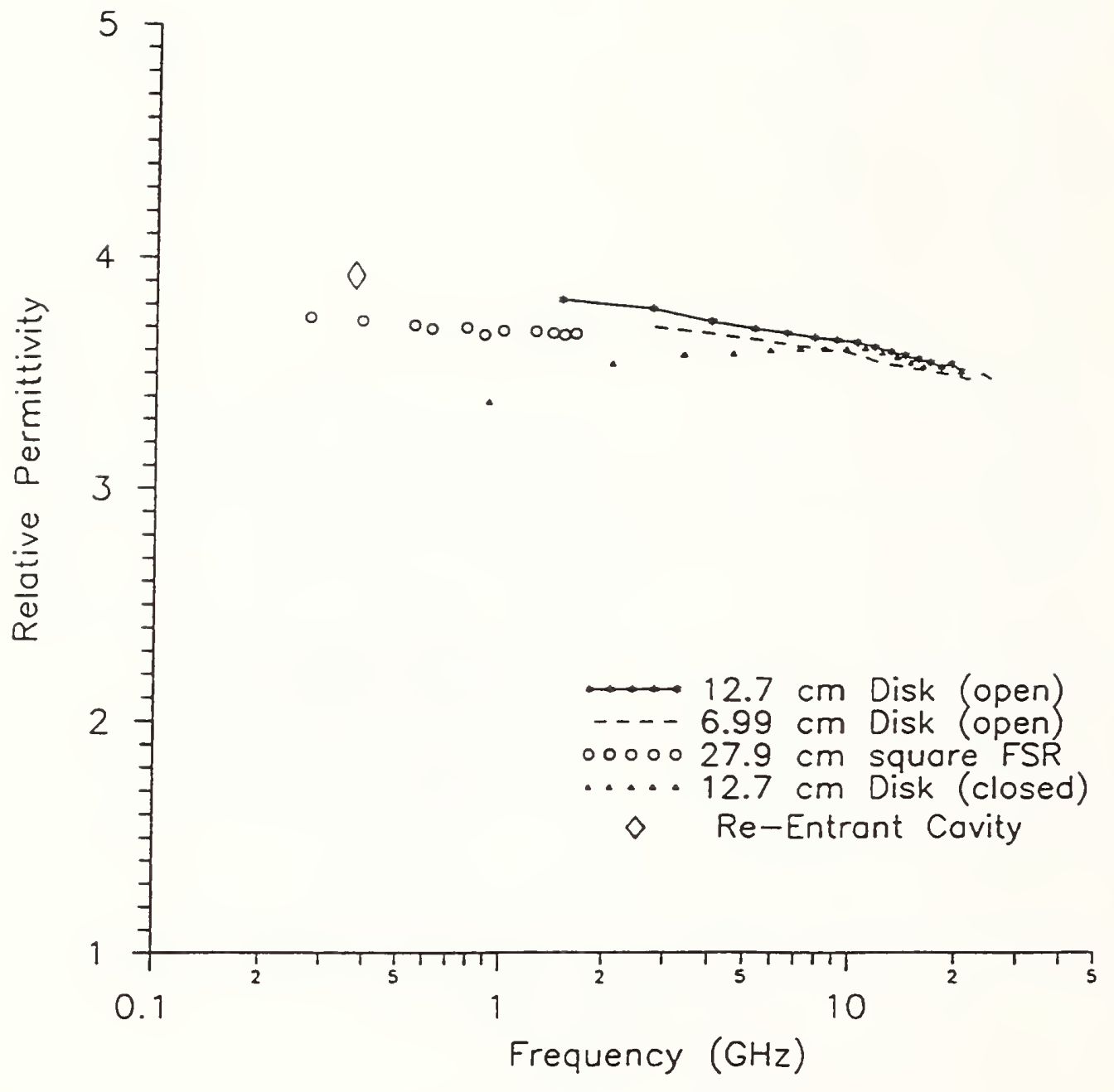

Figure 4. FSR permittivity results for $0.41 \mathrm{~mm}$ panel thick clad dielectric. 
exceptions, measured open disk quality factors were above 500 for the $6.99 \mathrm{~cm}$ disk and above 100 for the $12.7 \mathrm{~cm}$ disk. The vertical separation between the $12.7 \mathrm{~cm}$ disk and the $6.99 \mathrm{~cm}$ disk plots could be due to the smaller disk having more pronounced fringing error than the larger disk, say if the error varied as the ratio of the panel's perimeter to its area [5, fig. 5]. The re-entrant cavity result is seen to line up with the $12.7 \mathrm{~cm}$ circular disk results. Taking all this into account, our uncertainty estimate for the $12.7 \mathrm{~cm}$ open circular disk is $\pm 3 \%$.

\section{Conclusions and Recommendations}

We have provided expressions to improve FSR measurements and developed an uncertainty analysis for the FSR technique. With limited measurements, we have demonstrated that repeatable relative permittivity values are obtained using the FSR technique. Due to the potential for small deviation from a perfect side aspect ratio [5], square FSR panels can be more challenging to measure than rectangular ones. Software development is needed to obtain coupling coefficients using the ANA. As discussed in section 6, some difficulty was experienced obtaining quality factors greater than 50 with square FSR panels when the relative permittivity exceeded 2.5. Consequently, improved FSR coupling mechanisms need to be investigated, particularly mechanisms (such as described in [26]) enabling excitation along the side of a panel rather than just at a corner. Also, medium relative permittivity rectangular substrates need to be measured in order to further refine the uncertainty analysis.

Helpful comments and discussion with Claude Weil, James Baker-Jarvis, John Grosvenor, Michael Janezic, Chriss Jones, Richard Geyer, and Bill Riddle of the Dielectric-Material Properties group at NIST are gratefully acknowledged.

\section{REFERENCES}

[1] L. S. Napoli and J. J. Hughes, "A simple technique for the accurate determination of the microwave dielectric constant for microwave integrated circuit substrates," IEEE Trans. Microwave Theory Tech., vol. 19, pp. 664-665, July 1971.

[2] J. Q. Howell, "A quick accurate method to measure the dielectric constant of microwave integrated-circuit substrates," IEEE Trans. Microwave Theory Tech., vol. 21, pp. 142-143, March 1973.

[3] P. H. Ladbrooke, M. H. N. Potok, and E. H. England, "Coupling errors in cavity resonance measurements on MIC substrates," IEEE Trans. Microwave Theory Tech., vol. 21, pp. 560-562, Aug. 1973.

[4] G. R. Traut, "Modify test fixtures to determine PTFE dielectric constant," Microwaves and RF, vol. 27, pp. 115-124, Feb. 1988. 
[5] G. I. Woolaver, "Accurately measure dielectric constant of soft substrates," Microwaves and RF, vol. 29, pp. 153-158, Aug. 1990.

[6] J. D. Ryder, Networks, Lines, and Fields, second ed., Prentice Hall Inc., 1955, pp. 119-133.

[7] R. E. Collin, Foundations for Microwave Engineering, second ed., McGraw Hill Inc., 1992, pp. 525-545.

[8] D. Kajfez and P. Guillon, eds., Dielectric Resonators, Artech House Inc., 1986.

[9] E. F. Kuester, R. T. Johnk, and D. C. Chang, "The thin substrate approximation for reflection from the end of a slab-loaded parallel-plate waveguide with application to microstrip patch antennas," IEEE Trans. Antennas Propagat., vol. 30, no. 5, pp. 910-917, Sept. 1982.

[10] L. Lewin, Advanced Theory of Waveguides, "Wireless Engineer," Mliffe \& Sons, Ltd., 1951, chap. 6.

[11] E. L. Ginzton, Microwave Measurements, McGraw Hill Inc., 1957, pp. 396-409.

[12] D. Kajfez and E. J. Hwan, "Q factor measurement with network analyzer," IEEE Trans. Microwave Theory Tech., vol. 32, no. 7, pp. 666-670, July 1984.

[13] D. Kajfez, Q Factor, Vector Fields, Oxford, Miss., 1994.

[14] A. J. Estin and M. D. Janezic, "Improvements in dielectric measurements with a resonant cavity,” IEEE Inst. Meas. Conf. Rec., Atlanta, Ga., May 14-16, 1991.

[15] E. J. Vanzura and J. E. Rogers, "Resonant circuit model evaluation using reflected Sparameter data," IEEE Inst. Meas. Conf. Rec., Atlanta, Ga., May 14-16, 1991.

[16] R. C. Taber, "A parallel plate resonator technique for microwave loss measurements on superconductors," Rev. Sci. Instrum., vol. 61, no. 8, pp. 2200-2206, Aug. 1990.

[17] R. D. Lending, "New criteria for microwave component surfaces," Proc. Nat. Electronics Conf., vol. 11, pp. 391-401, 1955.

[18] D. C. Chang and E. F. Kuester, "Total and partial reflection from the end of a parallel plate waveguide with an extended dielectric slab," Radio Sci., vol. 16, no. 1, pp. 1-13, Jan.-Feb. 1981.

[19] A. Fathy, D. Kalokitis, and E. Belohoubek, "Microwave characteristics and characterization of high $\mathrm{T}_{\mathrm{c}}$ superconductors," Microwave J., vol. 31, no. 10, p. 75, Oct. 1988.

[20] J. V. L. Parry, "Measurement of permittivity and power factor of dielectrics at frequencies from 300 to 600 Mc/s," Proc. Inst. Elec. Engr., p. III, vol. 98, pp. 303-311, 1951. 
[21] B. N. Taylor and C. E. Kuyatt, "Guidelines for evaluating and expressing the uncertainty of NIST measurement results," Natl. Inst. Stand. Technol. Tech. Note 1297, Sept. 1994.

[22] ISO, "Guide to the expression of uncertainty in measurement," ISO Tech. Advisory Group, Geneva, Switzerland, 1993.

[23] J. R. Baker-Jarvis and B. F. Riddle, "Dielectric measurements using a reentrant cavity: Mode-matching analysis," Natl. Inst. Stand. Technol. Tech. Note 1384; 1997.

[24] C. N. Works, "Resonant cavities for dielectric measurements," J. Appl. Physics, vol. 18, pp. 605-612, July 1947.

[25] M. Abramowitz and I. A. Stegun, eds., Handbook of Mathematical Functions with Formulas, Graphs, and Mathematical Tables, Nat. Bur. Stand. (U.S.) App. Math Ser. 55, June 1964, chap. 9.

[26] "Non-destructive full sheet resonance test for permittivity of clad laminates," Inst. for Interconnecting and Packaging Electronic Circuits, IPC-TM-650 Test Methods Manual no. 2.5.5.6, May 1989. 


